\title{
Urinary Interleukin (Il)-18 as an Early Predictive Biomarker of Subclinical Proximal Tubular Dysfunction in HIV-Infected Patients Exposed to Tenofovir
}

\section{Orluwene CG ${ }^{1 *}$, Deebii ${ }^{2}$ and Odum EP1}

${ }^{1}$ Department of Chemical Pathology, University of Port Harcourt, Rivers State, Nigeria

${ }^{2}$ Department of Haematology, Blood Transfusion and Immunology, University of Port Harcourt, Rivers State, Nigeria

\begin{abstract}
Proximal tubular dysfunction (PTD) is a frequent complication of HIV-infected patients and lack of early biomarkers for PTD has impaired our ability to intervene in a timely manner. In this present study, we tested if interleukin- 18 (IL-18) is a predictive biomarker for PTD in HIV-infected patients on tenofovir (TDF) and Non-tenofovir (N-TDF) antiretroviral therapy and taking as control HIV treatment naïve patients. Exclusion criteria included pre-existing renal insufficiency and nephrotoxin used. Serial urine samples were analyzed by enzyme-linked immunosorbent assay for IL-18 in 254 HIV- infected patients at three different points (at baseline, at 4 weeks and at 12 weeks of fellow-up). Using eGFR values, marked decreased in kidney function was detected only at 12 weeks in the TDF regimen group $(p=0.003)$ as compared to other study groups. In contrast, urine IL-18 increased at a much early time (at 4 weeks) particularly in the TDF regimen group $(p=0.000)$ follow by the naïve group $(p=0.02)$ and continued to increase up to 12 weeks of follow up. This marked elevation is believed to be progressive. Our results indicate that IL-18 is an early, predictive biomarker of PTD and that this biomarker may allow for the reliable early diagnosis of PTD at all times in HIV-infected patients on TDF at risk of proximal tubular dysfunction, much before the rise in serum creatinine
\end{abstract}

\section{Keywords: TDF; HIV; IL-18; Nephrotoxicity}

\section{Introduction}

Interleukin (IL)-18 is an $18.3-\mathrm{kDa}$ cytokine with proinflammatory and immune-enhancing properties derived by enzymatic cleavage of a $23 \mathrm{kDa}$ precursor that is produced in monocytic cells. The pleiotropic activities of IL-18 suggest an important role of this cytokine in the triggering and polarization of the immune response. It has been found to be released by the proximal tubular cells of the kidney during the event of their damage by nephrotoxic drugs such as tenofovir (TDF) [1].

However, proximal tubular dysfuntion (PTD) is typically diagnosed by detecting increases in serum creatinine. Unfortunately, serum creatinine is an unreliable indicator during acute changes in kidney function owing to several reasons. First, serum creatinine concentrations might not change until about $50 \%$ of kidney function has already been lost. Second, serum creatinine does not accurately reflect kidney function until a steady state has been reached, which could take several days.

Finally, the serum levels of creatinine are affected by several non-renal factors such as age, diets, used of steroids, gender, race, and muscle mass [2]. All these reasons contribute to significant delays in the diagnosis of PTD. Also, the serum creatinine value at the time of diagnosis of PTD does not give any reliable information about prognosis of kidney toxicity as it does not reflect the severity of damage. However HIV infection and some antiretroviral (ARV) drugs have been implicated in the causation of renal impairment in humans. Tenofovir (TDF), a widely used first line antiretroviral drug have been widely reported to be associated with renal dysfunction [3,4]. Szczech et al. [4] have shown that TDF-associated nephrotoxicity is the most common single diagnose reason for HIVrelated referral to tertiary health services, accounting for about $20 \%$ of consultations. Alterations on tenofovir secretion by proximal renal tubule may lead directly to a greater drug accumulation in the renal tubular cells and, consequently lead to proximal tubular damage and renal toxicity [5-7].

\section{Material and Methods}

\section{Study population}

Two hundred and sixty seven HIV-infected naive patients were considered for participation in the study between Junes and November 2014. Thirteen patients were excluded because of nephrotoxic drugs use before recruitment. Thus, 254 patients were finally enrolled in the study: 100 TDF base regimen group and 102 Non-TDF regimen group While 52 HIV-treatment naïve patients served as potential controls. From every patient social demographics details of age, sex and occupation were recorded at the time of recruitment in to the study.

\section{Sample collection and analysis}

Blood serum samples were put in chemistry bottles while those for hematological analyses were collected using the EDTA bottles in order to avoid hemolytic reactions. The blood samples were then taken to the laboratory for various analyses.

Spot Urine samples were collected from patient's using urine sample bottle and analyzed within 4 hours of collection to avoid denaturation of some parameters. IL-18 was analyzed by enzymelinked immunosorbent assay (ELISA), while estimated glomerular filtration rate (eGFR) was calculated using the modification of diet in renal disease (MDRD) formula.

*Corresponding author: Orluwene CG, Department of Chemical Pathology, University of Port Harcourt, Rivers State, Nigeria, Tel: +2348033063385; E-mail: chituru.orluwene@uniport.edu.ng

Received July 17, 2014; Accepted August 24, 2015; Published September 07, 2015

Citation: Orluwene CG, Deebii N, Odum EP (2015) Urinary Interleukin (II)-18 as an Early Predictive Biomarker of Subclinical Proximal Tubular Dysfunction in HIV Infected Patients Exposed to Tenofovir. J AIDS Clin Res 6: 497. doi:10.4172/2155 6113.1000497

Copyright: (c) 2015 Orluwene CG, et al. This is an open-access article distributed under the terms of the Creative Commons Attribution License, which permits unrestricted use, distribution, and reproduction in any medium, provided the original author and source are credited. 
Citation: Orluwene CG, Deebii N, Odum EP (2015) Urinary Interleukin (II)-18 as an Early Predictive Biomarker of Subclinical Proximal Tubular Dysfunction in HIV-Infected Patients Exposed to Tenofovir. J AIDS Clin Res 6: 497. doi:10.4172/2155-6113.1000497

Page 2 of 4

\section{Statistical analysis}

Continuous values were given as mean and standard deviation while categorical data were given as percentages. The three groups of patients analyzed were first compared using chi-squared for categorical data and parametric tests for continuous variables. The level of significance was considered at $\mathrm{p}<0.05$. All statistical analyses were performed using SPSS version 20.0 software package (SPSS inc., Chicago, Illinois, USA). Carley et al. [8] simple normograms for the calculation of sample size for clinical diagnostic study was used for the calculation of sample size.

\section{Results}

A prospective observational study was started in June 2014 at the HIV clinic of University of Port Harcourt Teaching Hospital, a reference Hospital for tropical infectious disease located in Port Harcourt Rivers State. At recruitment, the patients were grouped as follows: $100 \mathrm{HIV}$ treatment Naïve patients about to start a TDF containing regimen (group 1), 102 HIV treatment Naïve patients about to start a NonTDF containing regimen (group 2) and While 52 HIV-treatment naïe patients served as potential controls (group 3).

\section{Characteristic of patients}

The results of the educational backgrounds of the study population were as follows: 6 (2.4\%) patients had no formal education, 27 (10.6\%) patients had primary education, $150(59.1 \%)$ patients had secondary education, $68(26.8 \%)$ patients had university education and $3(1.2 \%)$ patients $(\mathrm{p}>0.272)$ which was not statistically significant.

Among the study population, $53(20.9 \%)$ patients were jobless at present, $107(42.1 \%)$ were business men and women, $54(21.3 \%)$ were students, $26(10.2 \%)$ were artisans and only 14 (5.5\%) were civil servants. The difference among the groups was statistically significant $(\mathrm{P}>0.000)$.

The means age of the distinct patients' population; group 1, group 2 and group 3 are $35.23,35.49$ and 32.50 years respectively, but without a significant difference $(\mathrm{p}=0.069)$, although the gender distribution among the patients study population was statistically significant $(\mathrm{p}=0.002)$ and was observed to be; $85(33.46 \%)$ in males and 169 $(66.54 \%)$ in females as shown in Table 1.

Table 2 shows the eGFR of the study population at different points of the different regimen groups to be as follows: at baseline [TDF 107, $\mathrm{N}$-TDF 100.11, and Naïve, $110.25 \mathrm{mg} / \mathrm{dl}$ ], at 4 weeks [TDF 98.65, N-TDF 100.90, and Naïve, $107.63 \mathrm{mg} / \mathrm{dl}$ ] and at 12 weeks [TDF 75.63, $\mathrm{N}-\mathrm{TDF}$ 94.78, and Naïve, $80.54 \mathrm{mg} / \mathrm{dl}$ ]. The different between the groups was statistically significant only at 12 weeks of follow up.

\begin{tabular}{|c|c|c|c|c|c|}
\hline & Mean Age & Std. Deviation & No. of patient & P-I & Ialue \\
\hline SEXMale & 36.92 & 7.69 & $85(33.46 \%)$ & & \\
\hline Female & 33.70 & 7.97 & $169(66.54 \%)$ & (Z-test ) & $p=0.002$ \\
\hline Total & 34.77 & 8.01 & $254(100 \%)$ & & \\
\hline \multicolumn{6}{|c|}{ ART Regimen group } \\
\hline TDF & 35.23 & 7.68 & $100(39.37 \%)$ & & \\
\hline N-TDF & 35.49 & 8.68 & $102(40.16 \%)$ & (ANOVA) & $p=0.069$ \\
\hline D-NAIVE & 32.50 & 6.93 & $52(20.47 \%)$ & & \\
\hline Total & 34.77 & 8.02 & $254(100 \%)$ & & \\
\hline
\end{tabular}

Table 1: Age and Sex Characteristics of the study population.

\begin{tabular}{|c|c|c|c|c|c|}
\hline & HAART with TDF & HAART, Non-TDF & Drug-naïve & Difference among groups & Significance Difference among groups \\
\hline \multirow[t]{2}{*}{ No. of patience (\%) } & $100(39.37 \%)$ & $102(40.16 \%)$ & $52(20.47 \%)$ & & \\
\hline & Mean (S.D) & Mean (S.D) & Mean (S.D) & (ANOVA) P-value & (Z-Test) P-value \\
\hline eGFR at baseline $(\mathrm{mg} / \mathrm{dl})$ & $107.17(20.1)$ & $100.11(26.3)$ & $110.25(20.1)$ & $P=0.431$ & 1 versus $2 p=0.001,1$ versus $3 p=0.640,2$ versus $3 p=0.001$ \\
\hline eGFR at 4 weeks (mg/dl) & $98.65(19.1)$ & $100.90(19.4)$ & $107.63(25.4)$ & $P=0.416$ & $\begin{array}{c}1 \text { versus } 2 p=0.501,1 \text { versus } 3 p=0.475, \quad 2 \text { versus } 3 p= \\
0.600\end{array}$ \\
\hline eGFR at 12 weeks (mg/dl) & $75.63(15.4)$ & $94.78(16.3)$ & $80.54(13.4)$ & $P=0.031$ & $\begin{array}{l}1 \text { versus } 2 p=0.001,1 \text { versus } 3 p=0.003,2 \text { versus } 3 p= \\
0.997\end{array}$ \\
\hline
\end{tabular}

Values in parenthesis showed the standard error of mean

Table 2: Estimated glomerular filtration rate (eGFR) at different times point in the distinct patient populations

\begin{tabular}{|c|c|c|c|c|c|}
\hline & Drug Naïve (group 3) & N-TDF (group 2) & TDF (group 1) & Differences among groups & (Z-test) p-Value \\
\hline CD4 (ul/cells) & Means (S.D) & Means (S.D) & Means (S.D) & (ANOVA P Value) & 1 versus $2 p=0.000$ \\
\hline \multirow[t]{3}{*}{ VISIT 1} & \multirow[t]{3}{*}{$525.6(94.5)$} & \multirow[t]{3}{*}{$197.23(83.8)$} & \multirow[t]{3}{*}{$172.48(103.7)$} & \multirow[t]{3}{*}{0.000} & \\
\hline & & & & & 1 versus $3 p=0.000$ \\
\hline & & & & & 2 versus $3 p=0.613$ \\
\hline \multirow[t]{3}{*}{ VISIT 2} & \multirow[t]{3}{*}{$389.00(64.9)$} & \multirow[t]{3}{*}{$259.38(86.2)$} & \multirow[t]{3}{*}{$251.05(59.6)$} & \multirow[t]{3}{*}{0.000} & 1 versus $2 p=0.000$ \\
\hline & & & & & 1 versus $3 p=0.000$ \\
\hline & & & & & 2 versus $3 p=0.477$ \\
\hline \multirow[t]{3}{*}{ VISIT 3} & \multirow[t]{3}{*}{$276.60(51.6)$} & \multirow[t]{3}{*}{$363.24(131.1)$} & \multirow[t]{3}{*}{$659.95(109.0)$} & \multirow[t]{3}{*}{0.001} & 1 versus $2 p=0.002$ \\
\hline & & & & & 1 versus $3 p=0.006$, \\
\hline & & & & & 2 versus $3 p=0.043$ \\
\hline
\end{tabular}

Values in parenthesis shows the standard deviation

Table 3: Mean CD4 of the study population over time. 
The mean CD4 in all three groups increased progressively from visit 1 to visit 3, but the increase in TDF group double that in the N-TDF group and naïve group. The difference between the groups at each visit was statistically significant $(\mathrm{P}=0.000)$. The differences between the visits were observed to be; TDF [visit 1- 172.48, visit $2-251.05$, visit 3- 659.95], NTDF [visit 1- 197.23, visit 2- 259.38, visit 3- 363.24] and Naïve group [visit 1- 525.6, visit 2-389, visit 3- 276.6] (Table 3).

The minimum detectable dose of Human IL-18 in our laboratory was $15.6 \mathrm{pg} / \mathrm{ml}$. The kit recognized recombinant and natural Human IL-18 in urine sample. No significant cross-reactivity or interference was observed during analysis.

In the naïve group there was a progressive increase in the mean value of IL-18 at subsequent visits. It was as follows; $58.847 \mathrm{pg} / \mathrm{ml}$ (visit 1), $76.133 \mathrm{pg} / \mathrm{ml}$ (visit 2) and $96.873 \mathrm{pg} / \mathrm{ml}$ (visit 3). The difference between visit 1 and visit 3 was statistically significant (correlation coefficient $=0.577 \mathrm{P}=0.023$ ), but not between visit 1 and visit 2 and visit 2 and visit 3 (Figure 1).

In the TDF group there was also a progressive increase in the mean value of IL-18 at subsequent visits. The increase was much higher than that observed in the naïve group. The mean values at visit 1 , visit 2 and visit 3 were as follows; $64.905,88.386,107.74 \mathrm{pg} / \mathrm{ml}$ respectively. The differences among the visit were all statistically significant: visit 1 and visit 2 (correlation co-efficient $=0.93, \mathrm{P}=0.000$ ), Visit 1 and visit 3 (correlation co-efficient $=0.924, \mathrm{P}=0.000$ ), visit 2 and visit 3 (correlation co-efficient $=0.923, \mathrm{P}=0.000)$ (Figure 2).

However on the contrary, the N-TDF group showed a progressive decrease in the mean value of IL-18 at subsequent visits. The observed mean values at visit 1 , visit 2 and visit 3 were as follows; 48.929, 46.724, $46.019 \mathrm{pg} / \mathrm{ml}$ respectively. The differences between the values were not statistically significant as observed in the TDF group. The comparisons are: visit 1 and visit 2 (correlation co-efficient $=0.03, P=0.199$ ), visit 1 and visit 3 (correlation co-efficient $=0.305, P=0.192$ ), visit 2 and visit 3 (correlation co-efficient $=0.302, \mathrm{P}=0.180$ ) (Figure 3 ).

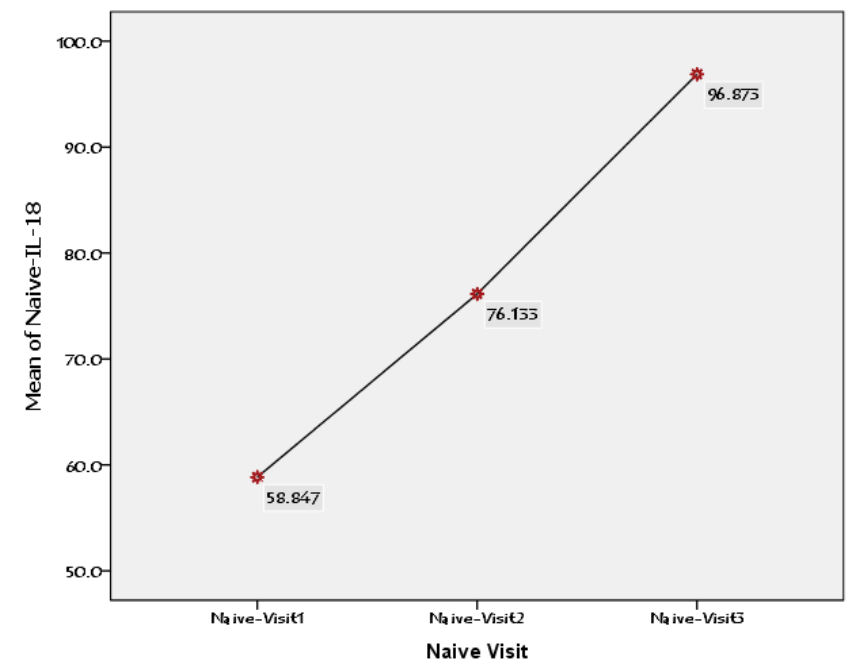

Figure 1: Changes in IL-18 Values in the Naive group with time

- Visit 1 (Commencement)

- Visit 2 after 4 Weeks

- Visit 3 after 12 weeks

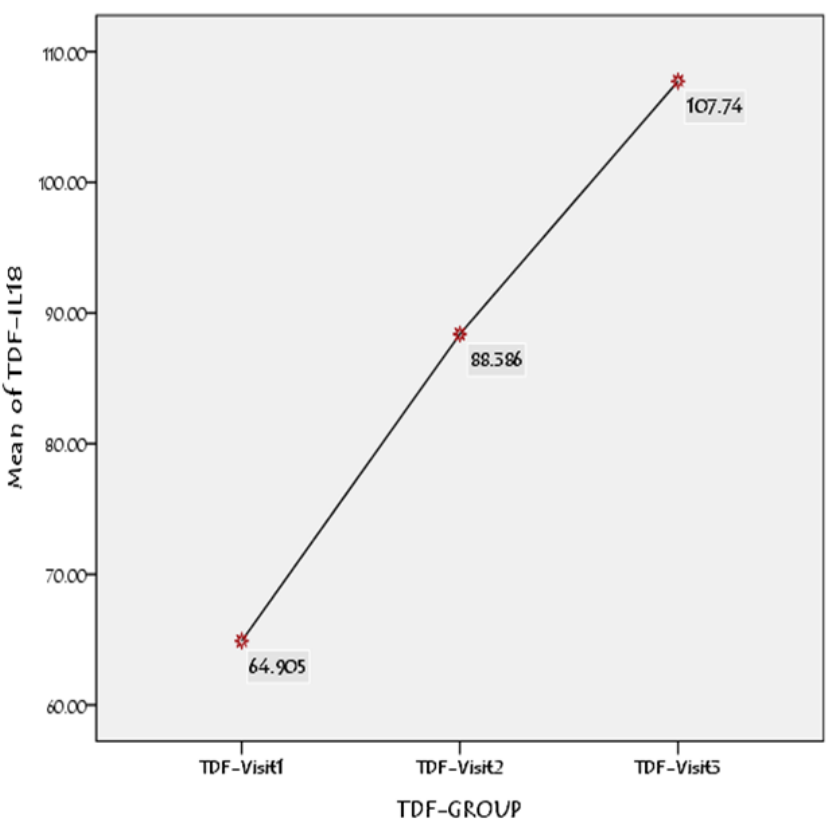

Figure 2: Changes in IL-18 Values in the TDF group with time

- Visit 1 (Commencement)

- Visit 2 after 4 Weeks

- Visit 3 after 12 weeks

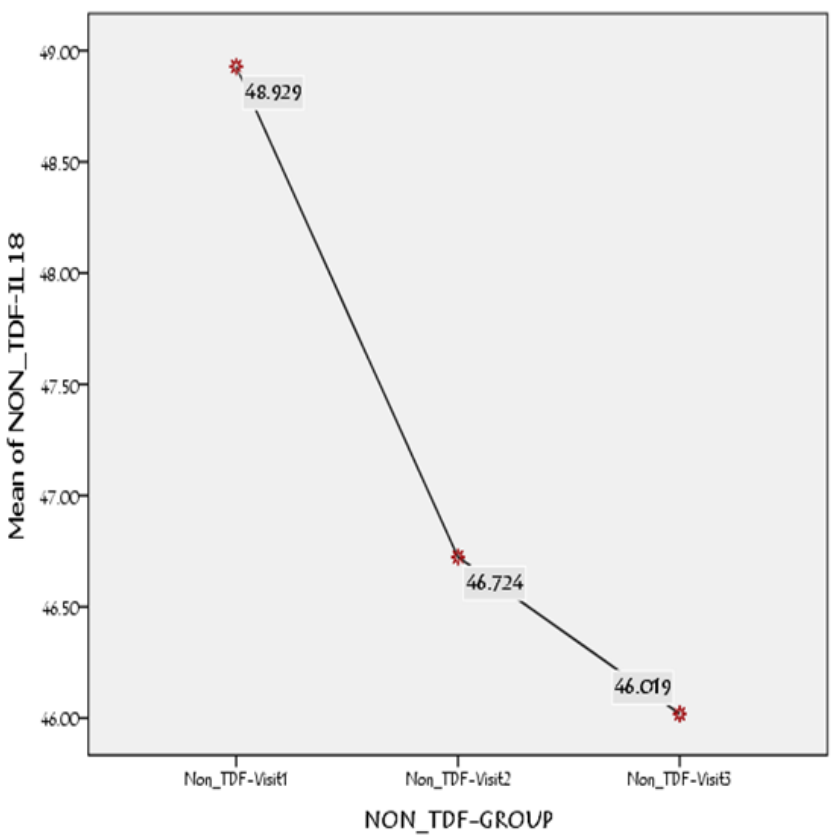

Figure 3: Changes in IL-18 Values in the N-TDF group with time

- Visit 1 (Commencement)

- Visit 2 after 4 Weeks

- Visit 3 after 12 weeks

\section{Discussion}

Interleukin - 18 is a proinflammatory cytokine produced by proximal tubular cells when damaged, $[9,10]$. Many investigators have analyzed urinary concentrations of IL-18 as an early markers of proximal renal injury. Their results suggest that IL-18 might be used as 
a marker to identify patients with proximal tubular dysfunction $[11,12]$. There are no reports on, studies to assess IL-18 value as a marker of proximal tubular disorder after months of exposure to TDF and other antiretroviral therapeutic agents in HIV-infected patients. The present study appears to be the first investigation in this area. Interleukin-18 has been described especially as a promising marker already in use to assess renal function after transplantations in metabolic diseases and heart diseases [10,13-15]. The results of the present study suggest that an increase in the urinary concentration of IL-18 followed the expression of classic laboratory features of renal insufficiency. IL-18 has also been reported to be a strong prognostic indicator of mortality in children treated in intensive care units [11]. Furthermore, Edelstein et al. [1] pointed out that IL-18 can also be a very useful predictive marker of renal insufficiency. In this study there was a statistically significant difference in IL-18 levels between each time points in the TDF regiment group, thus confirming earlier results from classic laboratory tests [15]. In this study Estimated glomerular filtration rate (eGFR) increased only slightly at 12 weeks of exposure particularly in the TDF regimen group pointing a delay in the detection of proximal tubular dysfunction compared to IL-18 that shows a marked increased at 4 weeks. There was also an increase in IL-18 levels with time, suggesting a possible progression of renal dysfunction from a subclinical stage to an end stage renal disease. It is therefore hypothesized that there might exist a possible relationship between nephrotoxicity caused by tenofovir and increased in IL-18 levels in HIV-infected patients on TDF first line antiretroviral therapy. In comparison with the $\mathrm{N}-\mathrm{TDF}$ regimen group, it was observed that IL-18 levels decreased sharply with time, suggesting that N-TDF antiretroviral therapy does not caused proximal tubular dysfunction but may offer remedy to the harmed caused by the HIV itself on the kidney. In this study no significant difference was observed between treatment points in these groups of patients, although this finding needs further elaborate study. This present study appear to confirm the hypothesis that IL-18 can be used as an early marker of proximal tubular dysfunction in HIV-infected patients treated with tenofovir and other nephrotoxic antiretroviral therapeutic agents.

\section{Conclusion}

In the patient population studied a clear pattern of IL-18 excretion was observed, with an increase in the TDF and Naive groups and fluctuating levels in the N-TDF group. The difference between the groups was highly statistically significant at different time point studied. Perhaps, this finding is expected since IL-18 increases in urine only under condition of a marked tubular damage, apoptotic tubular cell shedding, and cell necrosis, associated with deterioration of renal function [11]. From this study it was found that IL-18 seems to be a very reliable and accurate marker of subclinical nephropathy, especially tubulopathy in HIV-infected patients on TDF. This finding agrees with other workers that IL-18 might be used as a marker to identify patients with proximal tubular dysfunction $[11,12]$. Finally, the present findings further suggest that longer periods of observation may increase the number of patients with subclinical signs of nephropathy in patients exposed to TDF regimen since proximal tubular dysfunction in these patients is progressive.

\section{References}

1. Edelstein CL (2008) Biomarkers of acute kidney injury. Adv Chronic Kidney Dis 15: $222-234$

2. Jotwani V, Scherzer R, Abraham A, Estrella MM, Bennett M, et al. (2014) Does HIV infection promote early kidney injury in women? Antivir Ther 19: 79-87.

3. Ryom L, Mocroft A, Lundgren J (2012) HIV therapies and the kidney: some good, some not so good? Curr HIVIAIDS Rep 9: 111-120.

4. Szczech LA (2008) Renal dysfunction and tenofovir toxicity in HIV-infected patients. Top HIV Med 16: 122-126.

5. Rodriguez-Novoa S, Labarga P, Soriano V (2009) Predictors of kidney tubular dysfunction in HIV-infected patients treated with tenofovir: pharmacokinetics study. Clinical Infectious Disease 48: 108-116.

6. Rodríguez-Nóvoa S, Labarga $\mathrm{P}$, D'avolio A, Barreiro $\mathrm{P}$, Albalate $\mathrm{M}$, et al (2010) Impairment in kidney tubular function in patients receiving tenofovir is associated with higher tenofovir plasma concentrations. AIDS 24: 1064-1066.

7. Barrios A, García-Benayas T, González-Lahoz J, Soriano V (2004) Tenofovirrelated nephrotoxicity in HIV-infected patients. AIDS 18: 960-963.

8. Carley S, Dosman S, Jones SR, Harrison M (2005) Simple nomograms to calculate sample size in diagnostic studies. Emerg Med J 22: 180-181.

9. Ho E, Fard A, Maisel A (2010) Evolving use of biomarkers for kidney injury in acute care settings. Curr Opin Crit Care 16: 399-407.

10. Liang XL, Liu SX, Chen YH, Yan LJ, Li H, et al. (2010) Combination of urinary kidney injury molecule-1 and interleukin-18 as early biomarker for the diagnosis and progressive assessment of acute kidney injury following cardiopulmonary by-pass surgery: a prospective nested case control study. Biomarkers 15: 332-339.

11. Washburn KK, Zappitelli M, Arikan AA, Loftis L, Yalavarthy R, et al. (2008) Urinary interleukin-18 is an acute kidney injury biomarker in critically ill children. Nephrol Dial Transplant 23: 566-572.

12. Trof RJ, Di Maggio F, Leemreis J, Groeneveld AB (2006) Biomarkers of acute renal injury and renal failure. Shock 26: 245-253.

13. Rosner MH (2009) Urinary biomarkers for the detection of renal injury. Adv Clin Chem 49: 73-97.

14. Siew ED, Ikizler TA, Gebretsadik T, Shintani A, Wickersham N, et al. (2010) Elevated urinary IL-18 levels at the time of ICU admission predict adverse clinical outcomes. Clin J Am Soc Nephrol 5: 1497-1505.

15. Wu H, Craft ML, Wang P, Wyburn KR, Chen G, et al. (2008) IL-18 contributes to renal damage after ischemia-reperfusion. J Am Soc Nephrol 19: 2331-2341. 